\title{
Longitudinal Associations Between Glycemic Status and Cognitive Function in Older Participants at High Risk of Cardiovascular Disease: Two-Year Follow-Up in the PREDIMED-Plus Study
}

\section{Carlos Gómez Martínez}

Universitat Rovira i Virgili Facultat de Medicina I Ciences de la Salut

Nancy Babio

Universitat Rovira i Virgili Facultat de Medicina I Ciences de la Salut Jordi Julvez

IISPV: Institut d'Investigacio Sanitaria Pere Virgili

Nerea Becerra Tomás

Universitat Rovira i Virgili Facultat de Medicina I Ciences de la Salut

Miguel A. Martínez González

Universidad de Navarra

Dolores Corella

Universitat de Valencia

Olga Castañer

IMIM: Institut Hospital del Mar d'Investigacions Mediques

Dora Romaguera

Hospital Universitari Son Espases

Jesús Vioque

Miguel Hernandez University of Elche: Universidad Miguel Hernandez de Elche

Ángel M. Alonso-Gómez

Universidad del Pais Vasco

Julia Wärnberg

Universidad de Malaga

José A. Martínez

Universidad de Navarra

Luís Serra-Majem

Universidad de las Palmas de Gran Canaria

Ramón Estruch

Hospital Clinic de Barcelona

Francisco Tinahones

Universidad de Malaga 
José Lapetra

Distritio Sanitario Atención primaria Sevilla

\section{Xavier Pintó}

Bellvitge University Hospital: Hospital Universitari de Bellvitge

Josep A. Tur

Universitat de les Illes Balears

José López Miranda

IMIBIC: Instituto Maimonides de Investigacion Biomedica de Cordoba

\section{Aurora Bueno Cavanillas}

Universidad de Granada

José J. Gaforio

Universidad de Jaen

\section{Pilar Matía Martín}

Hospital Clinico Universitario San Carlos

\section{Lidia Daimiel}

IMDEA: Instituto Madrileno de Estudios Avanzados

\section{Vicente Martín Sánchez}

Universidad de Leon

\section{Josep Vidal}

Hospital Clinic de Barcelona

\section{Clotilde Vázquez}

Instituto de Investigacion Sanitaria de la Fundacion Jimenez Diaz

\section{Emilio Ros}

Hospital Clinic de Barcelona

\section{Søren Dalsgaard}

Aarhus University: Aarhus Universitet

\section{Carmen Sayón Orea}

Universidad de Navarra

José V. Sorlí

Universidad de Valencia: Universitat de Valencia

\section{Rafael de la Torre}

IMIM: Institut Hospital del Mar d'Investigacions Mediques

\section{Itziar Abete}

Universidad de Navarra

\section{Lucas Tojal Sierra}

Universidad del Pais Vasco

Francisco J. Barón López

Universidad de Malaga

\section{Noelia Fernández Brufal}

Centro de Salud San Fermín, Elche 
Jadwiga Konieczna

Hospital Universitari Son Espases

Antonio García Ríos

IMIBIC: Instituto Maimonides de Investigacion Biomedica de Cordoba

Emilio Sacanella

Hospital Clinic de Barcelona

\section{María R. Bernal López}

Universidad de Malaga

Jose M. Santos Lozano

Distritio Atención Primaria Sevilla

\section{Cristina Razquin}

Universidad de Navarra

\section{Andrea Álvarez Sala}

Universitat de Valencia

\section{Albert Goday}

IMIM: Institut Hospital del Mar d'Investigacions Mediques

\section{María de los A. Zulet}

Universidad de Navarra

\section{Jessica Vaquero Luna}

Universidad del Pais Vasco

Javier Diez Espino

Universidad de Navarra

\section{Aida Cuenca}

IMIM: Institut Hospital del Mar d'Investigacions Mediques

\section{Fernando Fernández Aranda}

Bellvitge University Hospital: Hospital Universitari de Bellvitge

\section{Mònica Bulló}

Universitat Rovira i Virgili Facultat de Medicina I Ciences de la Salut

Jordi Salas-Salvadó ( $\sim$ jordi.salas@urv.cat)

Universitat Rovira i Virgili Facultat de Medicina I Ciences de la Salut https://orcid.org/0000-0003-27007459

\section{Original investigation}

Keywords: cognitive function, diabetes duration, glycated hemoglobin, insulin resistance Type 2 Diabetes, prediabetes

Posted Date: August 12th, 2021

DOI: https://doi.org/10.21203/rs.3.rs-768645/v1 
License: (c) (1) This work is licensed under a Creative Commons Attribution 4.0 International License. Read Full License 


\section{Abstract}

BACKGROUND: Type 2 diabetes was related with larger cognitive decline. However, other glycemic dysregulations showed inconsistent results. Our aim was to examine longitudinal associations between diabetes/glycemic status and cognitive function in older adults with metabolic syndrome.

METHODS: We conducted a 2-year prospective cohort study $(n=6,874)$ within the framework of the PREDIMED-Plus study. The participants (with overweight/obesity and metabolic syndrome; mean age 64.9 years; $48.5 \%$ women) completed a battery of 8 cognitive tests, and a global cognitive function Zscore (GCF) was estimated. Participants were categorized by diabetes status (no-diabetes, prediabetes, and $<5$ or $\geq 5$-year diabetes duration), and control. At baseline, insulin resistance (HOMA-IR) and glycated hemoglobin $\left(\mathrm{HbA}_{1 \mathrm{c}}\right)$ levels were measured and antidiabetic medications were recorded. Linear and logistic regression models, adjusted by potential confounders, were fitted to assess associations between glycemic status and changes in cognitive function.

RESULTS: Prediabetes status was unrelated to cognitive decline. However, compared to participants without diabetes, those with $\geq 5$-year diabetes duration had greater reductions in the GCF $(\beta=-0.11[95 \% \mathrm{Cl}$ $-0.16 ;-0.06])$, processing speed and executive function measurements. Inverse associations were observed between baseline HOMA-IR and changes in the GCF ( $\beta=-0.0094$ [95\% $\mathrm{Cl}-0.0164 ;-0.0023])$, but also between $\mathrm{HbA}_{1 \mathrm{c}}$ levels and changes in the GCF $(\beta=-0.0610$ [95\% Cl $\left.-0.0889 ;-0.0331]\right)$, the Mini-Mental test, and other executive function tests. Poor diabetes control was inversely associated with phonologic fluency. Sulfonylureas, but especially insulin use, were related to cognitive decline.

CONCLUSIONS: Insulin resistance, diabetes status, longer diabetes duration, poor glycemic control, and insulin treatment were associated with worsening cognitive function at short-term in a population at high cardiovascular risk.

TRIAL REGISTRATION: ISRCTN, ISRCTN89898870. Registered 28 May 2014 - Retrospectively registered, http://www.isrctn.com/ISRCTN89898870

\section{Background}

Type 2 diabetes is an important public health problem worldwide. The International Diabetes Federation estimated that $\sim 463$ million people were living with diabetes in 2019 (and 374 million had prediabetes), of whom one-third were $>65$ years old, and this figure is expected to rise to 700 million by 2045 [1]. Diabetes mellitus is not only among the top 10 causes of death worldwide [2], but is also a risk factor for blindness, renal failure, and lower limb amputation, overall decreasing quality of life [2]. Meanwhile, over 50 million people worldwide live with dementia, and this number is expected to triple by 2050 [3]. Cognitive impairment, characterized by loss of memory, concentration and reduced ability to learn new things, affecting everyday life, is relatively common and is a costly condition for the health system [3]. 
Some meta-analyses and longitudinal studies of population-based cohorts show an increased risk of cognitive dysfunction in people with metabolic syndrome, prediabetes and diabetes [4-6]. Specifically, type 2 diabetes is related to deficits in different cognitive domains [7] and to accelerated cognitive decline, especially in psychomotor speed, memory and executive functions [8]. However, some prospective studies have failed to confirm these associations $[9,10]$. Also, the association between cognitive function/decline and metabolic syndrome, prediabetes, insulin resistance and glycemic control is less well understood $[4,6$, 11]. Therefore, more studies are warranted to determine if glycemic dysregulations before diabetes onset may have an effect on cognition in order to establish early strategies of prevention-focused on these populations.

The identification of risk factors for cognitive decline when type 2 diabetes has been already established is also of great interest because this could help screen individuals with diabetes who may particularly benefit from intensive and suitable treatment strategies. The risk of accelerated cognitive decline in type 2 diabetes has been reported by some studies to be dependent on both disease duration and glycemic control $[5,12]$. Glucose-lowering treatments have also been related to cognitive function/decline in few epidemiologic studies with moderate-quality evidence $[6,13]$. Therefore, more studies are required to increase the certainty of evidence of these associations.

The PREDIMED-Plus study offers an unprecedented opportunity to evaluate cognitive changes, using a battery of cognitive tests, by glycemic status in a large population with metabolic syndrome.

The objectives of the present study were to examine longitudinal associations between glycemic status (diabetes status, control/treatment, and related biomarkers) and cognitive decline and impairment. We hypothesized that glycemic dysregulations would be negatively associated with changes in cognitive function.

\section{Materials And Methods}

The present study is based on an observational prospective cohort design conducted within the framework of the PREDIMED-Plus study using 2 years of follow-up data. The PREDIMED-Plus study is a multicenter, randomized, parallel-group clinical trial conducted in Spain for primary cardiovascular disease prevention. Participants were randomized to an intensive weight loss intervention program based on an energy-restricted traditional Mediterranean diet, physical activity promotion and behavioral support (intervention group) or usual care consisting in general recommendations to follow an energy-unrestricted Mediterranean diet (control group). The study protocol has been described extensively elsewhere [14] and can be found in http://www.predimedplus.com. The trial was registered in 2014 at the International Standard Randomized Controlled Trial (http://www.isrctn.com/ISRCTN89898870).

\section{Study population}

Eligible participants were community-dwelling adults (55-75 years) with overweight/obesity ( $27 \leq \mathrm{BMI} \geq$ $40 \mathrm{~kg} / \mathrm{m} 2$ ) who met at least three criteria of metabolic syndrome [15]. Exclusion criteria are reported 
elsewhere [14].

Participant recruitment was conducted between October 2013 and December 2016 in 23 Spanish health centers. A total of 6,874 candidates met eligibility criteria and were randomly allocated in a $1: 1$ ratio to the intervention or control groups, using a centrally controlled, computer-generated random-number internetbased system with stratification by center, sex, and age. Couples sharing the same household were randomized together, using the couple as unit of randomization. The flow-chart of the studied PREDIMEDPlus population is shown in Additional file 1 (Additional file 1: Supplemental Fig. 1).

All participants provided written informed consent, and the study protocol and procedures were approved by all the ethical committees of all participating institutions.

\section{Diabetes status and glycemic measurements}

At baseline fasting blood samples were collected and biochemical analyses were performed to determine fasting plasma glucose and glycated hemoglobin ( $\mathrm{HbA1c}$ ) by routine laboratory methods. Insulin was centrally measured by an electrochemiluminescence immunoassay using an Elecsys immunoanalyzer (Roche Diagnostics, Meylan, France). Insulin resistance was estimated at baseline using the Homeostasis Model Assessment of Insulin Resistance (HOMA-IR) index [16].

Prediabetes and diabetes were defined following the American Diabetes Association criteria [17]. Diabetes was defined as previous diagnosis of diabetes, $\mathrm{HbA} 1 \mathrm{c} \geq 48 \mathrm{mmol} / \mathrm{mol}(6.5 \%)$, use of antidiabetic medication, or having fasting plasma glucose $>126 \mathrm{mg} / \mathrm{dl}$ in both the screening and baseline visits. Selfreported diabetes duration was categorized in $<5$-year and $\geq 5$-year diabetes duration. Prediabetes status was defined when $\mathrm{HbA} 1 \mathrm{c}$ was between $39 \mathrm{mmol} / \mathrm{mol}(5.7 \%)$ and $46 \mathrm{mmol} / \mathrm{mol}(6.4 \%)$, or having fasting plasma glucose between $\geq 100 \mathrm{mg} / \mathrm{dl}$ and $\leq 125 \mathrm{mg} / \mathrm{dl}$. Participants who did not meet any of these parameters were categorized into the no-diabetes category. Furthermore, we categorized diabetes status in participants presenting diabetes (participants with $<5$-year and $\geq 5$-year diabetes duration) and nodiabetes (participants with prediabetes and no-diabetes).

Glycated hemoglobin was used to categorize participants into those having good or poor diabetic control $(\mathrm{HbA} 1 \mathrm{c}<57 \mathrm{mmol} / \mathrm{mol}$ or $\geq 57 \mathrm{mmol} / \mathrm{mol}$ [7\%]), respectively [17]. Diabetes treatment was assessed at baseline using self-reported data on insulin, sulfonylureas, metformin or dipeptidyl peptidase-4 inhibitors (IDPP4) use.

\section{Covariates}

Covariates were evaluated at baseline by trained staff in a face-to-face interview using self-reported general questionnaires on socio-demographics (sex, age, level of education, and civil status), lifestyle (alcohol intake, smoking habits, physical activity, and Mediterranean diet adherence), and history of disease. Baseline anthropometric variables (weight and height) were determined to estimate body mass index (BMI). Adherence to an energy-reduced Mediterranean diet was assessed using a 17 food questionnaire, adapted from a previously validated one [18]. Leisure-time physical activity was estimated 
using a validated short version of the Minnesota Leisure-Time Physical Activity Questionnaire [19, 20]. Depressive status risk was evaluated using the Beck Depression Inventory-II [21].

\section{Neuropsychological assessment}

A battery of 8 cognitive tests was administered at baseline and 2 years of follow-up by trained staff. The tests performed, Mini-Mental State Examination (MMSE), Clock Drawing Test (CDT), Digit Span Test forward (DST-f) and backward (DST-b) section, Verbal Fluency Test animals (VFT-a) and "p" (VFT-p) version, and Trail Making Test part A (TMT-A) and B (TMT-B) are described in Additional file 1 (Additional file 1: Supplemental Material 1).

\section{Statistical analyses}

We used the December 2020 PREDIMED-Plus database. Descriptive variables are reported as means and standard deviation (SD) for continuous variables or numbers and percentages (\%) for qualitative variables. Differences between diabetes status and baseline characteristics were examined using chisquare and one-way ANOVA.

For longitudinal analysis, linear and logistic regression models were used, including only participants with complete cognitive data at baseline and 2 years of follow-up for each cognitive test analyzed. To facilitate comparisons across cognitive tests, Z-scores were generated for each cognitive score at baseline and after 2 years using the mean and SD of baseline data, as previously reported [5, 12]. A global cognitive function Z-score (GCF) was obtained averaging all cognitive Z-scores at each time point, standardizing by the mean and SD of cognitive Z-scores at baseline.

Using linear regression analyses we examined the associations between baseline status and 2-year changes in cognitive Z-scores in relation to: a) HOMA-IR levels; b) diabetes status, no diabetes being the reference group; c) HbA1c levels; d) glycemic control measured by HbA1c in participants with diabetes, good glycemic control being the reference group; e) diabetes treatment in participants with diabetes, no treatment being the reference group. Two models were fitted to adjust linear and logistic regression analyses. Model 1 was adjusted for sex, age (years), intervention group, and center size (with < 250; 250300, 300-400; >400 randomized participants). Model 2 was additionally adjusted for education level (primary school; high school; college), civil status (single, divorced or separated; married; widower), physical activity (MET min/week), smoking habits (smoker; former smoker; never smoker), alcohol intake (g/day), 17-point Mediterranean diet score, BMI (kg/m2), hypertension (yes/no), hypercholesterolemia (yes/no), and depression (yes/no).

Logistic regression analyses were used to estimate odds ratios (OR) and 95\% confidence intervals $(95 \% \mathrm{Cl})$, examining the 2-year risk for cognitive impairment in participants with normal cognitive performance at baseline by diabetes status, no diabetes being the reference group. Cognitive function cutoffs were defined by the dichotomization of neuropsychological assessments at the respective visits. Cognitive impairment was defined as GCF $\leq 10$ th percentile, MMSE $\leq 24$ punctuation, CDT $\leq 4$ 
punctuation, and VFT-a, VFT-p, DST-d, DST-b $\leq$ respective mean $-1.5 S D$ and TMT-A, TMT-B $\geq$ respective mean + 1.5SD [22-25].

Interaction analyses between glycemic status (diabetes status, HOMA-IR, HbA1c, and glycemic control and treatment) and sex, age, hypertension and BMI for the GCF were performed by comparing the model with and without the interaction product using the likelihood ratio test.

Sensitivity analyses were performed to assess the associations between diabetes treatment and cognitive assessments controlling by diabetes duration or glycemic control. Participants with missing data on covariables (always $<1 \%$ missings) were imputed as either the mean of the group or into the subcategory with the highest frequency [26].

All analyses were conducted with robust estimates of the variance to correct for intracluster correlation. The data were analyzed using the Stata-14 software program (StataCorp), and statistical significance was set using the Benjamini-Hochberg false discovery rate correction procedure [27] at a P-value $<0.05$.

\section{Results}

\section{Descriptive results}

Table 1 shows the baseline characteristics of the study population ( $n=6,874)$ according to diabetes status. A total of $20.9 \%$ of participants were classified having no-diabetes, $48.6 \%$ prediabetes, $14.8 \%$ with $<5$-year diabetes duration, and $15.6 \%$ with $\geq 5$-year diabetes duration. The mean age of the total population was $64.9 \pm 4.9$ years and $48.5 \%$ were women. Participants with $\geq 5$-year diabetes duration were older, had lower educational level and alcohol consumption, higher adherence to the Mediterranean diet and high $\mathrm{HbA} 1 \mathrm{c}$ levels. They were also more likely to have hypertension, hypercholesterolemia and depressive symptoms. Participants with < 5-year diabetes duration had higher obesity prevalence and HOMA-IR levels, and were less likely to be women. Participants without diabetes were more likely to have a higher educational level. All cognitive assessments showed significant differences across diabetes status and participants with $\geq$ 5-year diabetes duration presented lower scores. 
Table 1

Baseline characteristics by diabetes status

\begin{tabular}{|c|c|c|c|c|c|}
\hline \multirow[t]{2}{*}{ Characteristics } & \multicolumn{4}{|c|}{ Diabetes status } & \multirow{2}{*}{$\begin{array}{l}\mathrm{P} \text { - } \\
\text { value }\end{array}$} \\
\hline & $\begin{array}{l}\text { No- } \\
\text { Diabetes } \\
(n= \\
1440)\end{array}$ & $\begin{array}{l}\text { Prediabetes } \\
(n=3341)\end{array}$ & $\begin{array}{l}<5 y \text { Diabetes } \\
(n=1020)\end{array}$ & $\begin{array}{l}\geq 5 y \text { Diabetes } \\
(n=1073)\end{array}$ & \\
\hline Age (years) & $\begin{array}{l}64.5 \pm \\
4.92\end{array}$ & $65.0 \pm 4.91$ & $64.7 \pm 4.98$ & $65.5 \pm 4.81$ & $\begin{array}{l}< \\
0.001\end{array}$ \\
\hline Sex (women) & $\begin{array}{l}706 \\
(49.03)\end{array}$ & 1703 (50.97) & $435(42.65)$ & $491(45.76)$ & $<.001$ \\
\hline Intervention group & $\begin{array}{l}730 \\
(50.69)\end{array}$ & $1632(48.85)$ & $503(49.31)$ & $541(50.42)$ & 0.623 \\
\hline Education level & & & & & $\begin{array}{l}< \\
0.001\end{array}$ \\
\hline $\begin{array}{l}\text { Primary school or } \\
\text { less }\end{array}$ & $\begin{array}{l}653 \\
(45.35)\end{array}$ & $1627(48.70)$ & $489(47.94)$ & $593(55.27)$ & \\
\hline High school & $\begin{array}{l}417 \\
(28.96)\end{array}$ & $976(29.21)$ & $302(29.61)$ & $291(27.12)$ & \\
\hline College & $\begin{array}{l}370 \\
(25.69)\end{array}$ & 738 (22.09) & $229(22.45)$ & 189 (17.61) & \\
\hline Civil status & & & & & 0.803 \\
\hline $\begin{array}{l}\text { Single, divorced or } \\
\text { separated }\end{array}$ & $\begin{array}{l}199 \\
(13.82)\end{array}$ & $440(13.17)$ & $123(12.06)$ & $135(12.58)$ & \\
\hline Married & $\begin{array}{l}1097 \\
(76.18)\end{array}$ & $2546(76.20)$ & 797 (78.14) & $821(76.51)$ & \\
\hline Widower & $\begin{array}{l}144 \\
(10.00)\end{array}$ & $355(10.63)$ & $100(9.80)$ & $117(10.90)$ & \\
\hline
\end{tabular}

Abbreviations: $<5 y$ diabetes, less than 5 years diabetes duration; $\geq 5 y$ diabetes, more than 5 years diabetes duration; GCF, Global Cognitive Function; MMSE, Mini-Mental State Examination; CDT, Clock Drawing Test; DST-f, Digit Span Test forward section; DST-b, Digit Span Test backward section; VFT-a, Verbal Fluency Test animal category; VFT-p, Verbal Fluency Test letter "p"; TMT-A, Trail Making Test part A; TMT-B, Trail Making Test part B

$\S$ Inverse neuropsychological assessment score

Data are $\mathrm{n}(\%)$ or mean \pm SD for categorical and quantitative variables, respectively

Only the participants reported in each neuropsychological assessment are available

Chi-square is used for categorical variables and One-way ANOVA for quantitative variables 


\begin{tabular}{|c|c|c|c|c|c|}
\hline \multirow[t]{2}{*}{ Characteristics } & \multicolumn{4}{|c|}{ Diabetes status } & \multirow{2}{*}{$\begin{array}{l}\mathrm{P} \text { - } \\
\text { value }\end{array}$} \\
\hline & $\begin{array}{l}\text { No- } \\
\text { Diabetes } \\
(n= \\
1440)\end{array}$ & $\begin{array}{l}\text { Prediabetes } \\
(n=3341)\end{array}$ & $\begin{array}{l}<5 y \text { Diabetes } \\
(n=1020)\end{array}$ & $\begin{array}{l}\geq 5 y \text { Diabetes } \\
(n=1073)\end{array}$ & \\
\hline Physical activity & $\begin{array}{l}2508 \pm \\
2433\end{array}$ & $2493 \pm 2264$ & $2344 \pm 2140$ & $2420 \pm 2378$ & 0.236 \\
\hline \multicolumn{6}{|l|}{ (MET min/week) } \\
\hline Current smoker & & & & & 0.195 \\
\hline Smoker & $\begin{array}{l}170 \\
(11.81)\end{array}$ & $418(12.51)$ & $138(13.53)$ & $131(12.21)$ & \\
\hline Former smoker & $\begin{array}{l}602 \\
(41.81)\end{array}$ & 1434 (42.92) & 463 (45.39) & $484(45.11)$ & \\
\hline Never smoker & $\begin{array}{l}668 \\
(46.39)\end{array}$ & $1434(44.57)$ & $419(41.08)$ & $458(42.68)$ & \\
\hline \multicolumn{6}{|c|}{$\begin{array}{l}\text { Abbreviations: <5y diabetes, less than } 5 \text { years diabetes duration; } \geq 5 y \text { diabetes, more than } 5 \text { years } \\
\text { diabetes duration; GCF, Global Cognitive Function; MMSE, Mini-Mental State Examination; CDT, Clock } \\
\text { Drawing Test; DST-f, Digit Span Test forward section; DST-b, Digit Span Test backward section; VFT-a, } \\
\text { Verbal Fluency Test animal category; VFT-p, Verbal Fluency Test letter "p"; TMT-A, Trail Making Test } \\
\text { part A; TMT-B, Trail Making Test part B }\end{array}$} \\
\hline \multicolumn{6}{|c|}{$\S$ Inverse neuropsychological assessment score } \\
\hline \multicolumn{6}{|c|}{ Data are $\mathrm{n}(\%)$ or mean \pm SD for categorical and quantitative variables, respectively } \\
\hline \multicolumn{6}{|c|}{ Only the participants reported in each neuropsychological assessment are available } \\
\hline
\end{tabular}




$\begin{array}{llll}\begin{array}{l}\text { No- } \\ \text { Diabetes }\end{array} & \text { Prediabetes } & <5 y \text { Diabetes } & \geq 5 y \text { Diabetes } \\ (n= & (n=3341) & (n=1020) & (n=1073) \\ 1440) & & & \end{array}$

\begin{tabular}{|c|c|c|c|c|c|}
\hline $\begin{array}{l}\text { Alcohol consumption } \\
\text { (g/day) }\end{array}$ & $\begin{array}{l}11.0 \pm \\
14.2\end{array}$ & $11.6 \pm 15.9$ & $11.7 \pm 15.6$ & $9.8 \pm 14.6$ & 0.004 \\
\hline $\begin{array}{l}\text { 17-point } \\
\text { Mediterranean diet } \\
\text { score }\end{array}$ & $\begin{array}{l}8.51 \pm \\
2.71\end{array}$ & $8.37 \pm 2.70$ & $8.64 \pm 2.60$ & $8.72 \pm 2.55$ & 0.001 \\
\hline $\mathrm{BMI}\left(\mathrm{kg} / \mathrm{m}^{2}\right)$ & $\begin{array}{l}32.2 \pm \\
3.46\end{array}$ & $32.6 \pm 3.41$ & $32.9 \pm 3.49$ & $32.6 \pm 3.52$ & $\begin{array}{l}< \\
0.001\end{array}$ \\
\hline HOMA-IR & $\begin{array}{l}3.91 \pm \\
2.61\end{array}$ & $5.08 \pm 3.14$ & $6.65 \pm 4.19$ & $6.30 \pm 4.45$ & $\begin{array}{l}<.001 \\
0.001\end{array}$ \\
\hline
\end{tabular}

Abbreviations: $<5 y$ diabetes, less than 5 years diabetes duration; $\geq 5 y$ diabetes, more than 5 years diabetes duration; GCF, Global Cognitive Function; MMSE, Mini-Mental State Examination; CDT, Clock Drawing Test; DST-f, Digit Span Test forward section; DST-b, Digit Span Test backward section; VFT-a, Verbal Fluency Test animal category; VFT-p, Verbal Fluency Test letter "p"; TMT-A, Trail Making Test part A; TMT-B, Trail Making Test part B

$\S$ Inverse neuropsychological assessment score

Data are $\mathrm{n}(\%)$ or mean \pm SD for categorical and quantitative variables, respectively

Only the participants reported in each neuropsychological assessment are available

Chi-square is used for categorical variables and One-way ANOVA for quantitative variables 


$\begin{array}{llll}\text { No- } & \text { Prediabetes } & <5 y \text { Diabetes } & \geq 5 y \text { Diabetes } \\ \text { Diabetes } & (n=3341) & (n=1020) & (n=1073) \\ (n= & & & \\ 1440) & & & \end{array}$

\begin{tabular}{lllccl}
$\mathrm{HbA}_{1 \mathrm{c}}(\mathrm{mmol} / \mathrm{mol})$ & $\begin{array}{l}36.4 \pm \\
4.7\end{array}$ & $40.5 \pm 3.5$ & $49.3 \pm 10.2$ & $54.7 \pm 13.1$ & $<$ \\
\hline $\mathrm{HbA}_{1 \mathrm{c}}(\%)$ & $\begin{array}{l}5.48 \pm \\
0.43\end{array}$ & $5.86 \pm 0.32$ & $6.66 \pm 0.93$ & $7.16 \pm 1.20$ & $<$ \\
\hline Hypertension & $\begin{array}{l}1192 \\
(82.78)\end{array}$ & $2764(82.73)$ & $855(83.82)$ & $947(88.26)$ & $<$ \\
\hline Hypercholesterolemia & $\begin{array}{l}966 \\
(67.08)\end{array}$ & $2281(68.27)$ & $755(74.02)$ & $811(75.58)$ & $<$ \\
\hline $\begin{array}{l}\text { Depressive } \\
\text { symptomatology }\end{array}$ & $\begin{array}{l}281 \\
(19.51)\end{array}$ & $667(19.96)$ & $226(22.16)$ & $253(23.58)$ & 0.001 \\
\hline
\end{tabular}

\section{Cognitive} assessments

\section{Diabetes status}

No-Diabetes Prediabetes

$28.3 \pm 1.85$

$28.3 \pm 1.86$

$5.96 \pm 1.21$

$5.95 \pm 1.29$

$8.95 \pm 2.59$

$8.78 \pm 2.39$

$5.11 \pm 2.20$

$5.28 \pm 2.36$

$16.4 \pm 5.00$
$16.1 \pm 4.75$
$8.87 \pm 2.48$

$<5 y$ Diabetes

$28.2 \pm 1.95$

$6.02 \pm 1.12$

$8.87 \pm 2.48$

$5.19 \pm 2.19$

$16.1 \pm 4.84$

\section{$\geq 5 y$}

Diabetes

$\begin{array}{ll}28 \pm & < \\ 2.10 & 0.001\end{array}$

VFT-a $(n=6816)$

$\begin{array}{ll}5.76 \pm & < \\ 1.34 & 0.00\end{array}$

0.001

$\begin{array}{ll}8.52 \pm & < \\ 2.48 & 0.001\end{array}$

DST-b $(n=5864)$

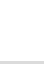




\begin{tabular}{|c|c|c|c|c|c|}
\hline \multirow[t]{2}{*}{ Characteristics } & \multicolumn{4}{|c|}{ Diabetes status } & \multirow{2}{*}{$\begin{array}{l}\mathrm{P}- \\
\text { value }\end{array}$} \\
\hline & $\begin{array}{l}\text { No- } \\
\text { Diabetes } \\
(n= \\
1440)\end{array}$ & $\begin{array}{l}\text { Prediabetes } \\
(n=3341)\end{array}$ & $\begin{array}{l}<5 y \text { Diabetes } \\
(n=1020)\end{array}$ & $\begin{array}{l}\geq 5 y \text { Diabetes } \\
(n=1073)\end{array}$ & \\
\hline VFT-p $(n=6816)$ & $12.6 \pm 4.62$ & $12.4 \pm 4.53$ & $12 \pm 4.35$ & $\begin{array}{l}11.4 \pm \\
4.39\end{array}$ & $\begin{array}{l}< \\
0.001\end{array}$ \\
\hline TMT-A $(n=6802) \S$ & $50.9 \pm 28.0$ & $52.3 \pm 27.5$ & $52.7 \pm 30.2$ & $\begin{array}{l}56.2 \pm \\
30.2\end{array}$ & $\begin{array}{l}< \\
0.001\end{array}$ \\
\hline TMT-B $(n=6784) \S$ & $121.6 \pm 68.6$ & $128.0 \pm 70.2$ & $130.1 \pm 72.3$ & $\begin{array}{l}144.2 \pm \\
79.6\end{array}$ & $\begin{array}{l}<.001 \\
0.001\end{array}$ \\
\hline \multicolumn{6}{|c|}{$\begin{array}{l}\text { Abbreviations: }<5 y \text { diabetes, less than } 5 \text { years diabetes duration; } \geq 5 y \text { diabetes, more than } 5 \text { years } \\
\text { diabetes duration; GCF, Global Cognitive Function; MMSE, Mini-Mental State Examination; CDT, Clock } \\
\text { Drawing Test; DST-f, Digit Span Test forward section; DST-b, Digit Span Test backward section; VFT-a, } \\
\text { Verbal Fluency Test animal category; VFT-p, Verbal Fluency Test letter "p"; TMT-A, Trail Making Test } \\
\text { part A; TMT-B, Trail Making Test part B }\end{array}$} \\
\hline \multicolumn{6}{|c|}{$\S$ Inverse neuropsychological assessment score } \\
\hline \multicolumn{6}{|c|}{ Data are $n(\%)$ or mean \pm SD for categorical and quantitative variables, respectively } \\
\hline \multicolumn{6}{|c|}{ Only the participants reported in each neuropsychological assessment are available } \\
\hline
\end{tabular}

Additional file 1 shows the flow of participants included in each analysis (Additional file 1: Supplemental Fig. 1).

\section{Diabetes status and related biomarkers}

Table 2 shows the associations between baseline diabetes status and 2-year changes in cognitive Zscores. No significant differences in the associations between participants with and without diabetes were observed in relation to cognitive tests used. Compared to participants without diabetes, those with < 5-year diabetes duration displayed higher decrements in cognitive Z-scores measured by the GCF, VFT-a, VFT-p and TMT-B tests in model 1, however, these associations disappear in model 2. Compared to participants without diabetes, those with $\geq 5$-year diabetes duration displayed higher reductions in all cognitive assessments in model 1, except in case of the CDT test (Table 2). However, in model 2 these associations remained significant only for GCF score, and the VFT-a, VFT-p, TMT-A and TMT-B tests. Similar results were found comparing participants with diabetes and no-diabetes. Compared to those without diabetes, participants with diabetes showed a higher 2-year decrease in the MMSE score in model 2 (Additional file 1: Supplemental Table 1). 
Table 2

Association between baseline diabetes status and changes in cognitive Z-scores

\begin{tabular}{|c|c|c|c|c|c|}
\hline \multirow{2}{*}{$\begin{array}{l}\text { Z- } \\
\text { scores }\end{array}$} & \multirow[t]{2}{*}{ Diabetes status } & \multicolumn{2}{|l|}{ Model 1} & \multicolumn{2}{|l|}{ Model 2} \\
\hline & & $\beta(95 \% \mathrm{Cl})$ & P-value & $\beta(95 \% \mathrm{Cl})$ & P-value \\
\hline \multirow[t]{4}{*}{ GCF } & $\begin{array}{l}\text { No-Diabetes }(n= \\
1023)\end{array}$ & Ref. & & Ref. & \\
\hline & Prediabetes $(n=2429)$ & $\begin{array}{l}-0.04(-0.10 \\
0.03)\end{array}$ & 0.277 & $\begin{array}{l}-0.01(-0.04 \\
0.03)\end{array}$ & 0.756 \\
\hline & $\begin{array}{l}<5 y \text { Diabetes }(n= \\
667)\end{array}$ & $\begin{array}{l}-0.12(-0.20 \\
-0.03)\end{array}$ & $0.008^{*}$ & $\begin{array}{l}-0.04(-0.09 \\
0.01)\end{array}$ & 0.109 \\
\hline & $\begin{array}{l}\geq 5 y \text { Diabetes }(n= \\
684)\end{array}$ & $\begin{array}{l}-0.27(-0.36 \\
-0.18)\end{array}$ & $\dot{<} 0.001 *$ & $\begin{array}{l}-0.11(-0.16 \\
-0.06)\end{array}$ & $<0.001^{*}$ \\
\hline \multirow[t]{4}{*}{ MMSE } & $\begin{array}{l}\text { No-Diabetes }(n= \\
1187)\end{array}$ & Ref. & & Ref. & \\
\hline & Prediabetes $(n=2786)$ & $\begin{array}{l}-0.01(-0.07 \\
0.05)\end{array}$ & 0.749 & $0.01(-0.05,0.06)$ & 0.865 \\
\hline & $\begin{array}{l}<5 y \text { Diabetes }(n= \\
847)\end{array}$ & $\begin{array}{l}-0.08(-0.16 \\
0.01)\end{array}$ & 0.054 & $\begin{array}{l}-0.05(-0.13 \\
0.03)\end{array}$ & 0.209 \\
\hline & $\begin{array}{l}\geq 5 y \text { Diabetes }(n= \\
865)\end{array}$ & $\begin{array}{l}-0.11(-0.19 \\
-0.02)\end{array}$ & $0.011^{*}$ & $\begin{array}{l}-0.06(-0.14 \\
0.02)\end{array}$ & 0.134 \\
\hline \multirow[t]{4}{*}{ CDT } & $\begin{array}{l}\text { No-Diabetes }(\mathrm{n}= \\
1189)\end{array}$ & Ref. & & Ref. & \\
\hline & Prediabetes $(n=2788)$ & $0.01(-0.06,0.07)$ & 0.874 & $0.01(-0.05,0.07)$ & 0.780 \\
\hline & $\begin{array}{l}<5 y \text { Diabetes }(n= \\
846)\end{array}$ & $\begin{array}{l}-0.01(-0.09 \\
0.08)\end{array}$ & 0.843 & $0.01(-0.08,0.09)$ & 0.847 \\
\hline & $\begin{array}{l}\geq 5 y \text { Diabetes }(n= \\
866)\end{array}$ & $\begin{array}{l}-0.09(-0.18 \\
-0.01)\end{array}$ & 0.031 & $\begin{array}{l}-0.06(-0.14 \\
0.03)\end{array}$ & 0.171 \\
\hline \multirow[t]{4}{*}{ DST-f } & $\begin{array}{l}\text { No-Diabetes }(n= \\
1072)\end{array}$ & Ref. & & Ref. & \\
\hline & Prediabetes $(n=2526)$ & $\begin{array}{l}-0.03(-0.10 \\
0.05)\end{array}$ & 0.474 & $\begin{array}{l}-0.01(-0.08 \\
0.06)\end{array}$ & 0.725 \\
\hline & $\begin{array}{l}<5 y \text { Diabetes }(n= \\
702)\end{array}$ & $\begin{array}{l}-0.08(-0.17 \\
0.01)\end{array}$ & 0.087 & $\begin{array}{l}-0.06(-0.15 \\
0.03)\end{array}$ & 0.198 \\
\hline & $\begin{array}{l}\text { Z5y Diabetes }(n= \\
716)\end{array}$ & $\begin{array}{l}-0.12(-0.21 \\
-0.03)\end{array}$ & $0.012^{\star}$ & $\begin{array}{l}-0.07(-0.16 \\
0.02)\end{array}$ & 0.126 \\
\hline \multirow[t]{2}{*}{ DST-b } & $\begin{array}{l}\text { No-Diabetes }(n= \\
1072)\end{array}$ & Ref. & & Ref. & \\
\hline & Prediabetes $(n=2525)$ & $\begin{array}{l}-0.04(-0.11 \\
0.03)\end{array}$ & 0.293 & $\begin{array}{l}-0.02(-0.09 \\
0.04)\end{array}$ & 0.528 \\
\hline
\end{tabular}




\begin{tabular}{|c|c|c|c|c|c|}
\hline & $\begin{array}{l}<5 y \text { Diabetes }(n= \\
702)\end{array}$ & $\begin{array}{l}-0.07(-0.16 \\
0.02)\end{array}$ & 0.116 & $\begin{array}{l}-0.04(-0.13 \\
0.04)\end{array}$ & 0.349 \\
\hline & $\begin{array}{l}\text { 716y Diabetes }(n= \\
\text { (1) }\end{array}$ & $\begin{array}{l}-0.11(-0.20 \\
-0.02)\end{array}$ & $0.014^{*}$ & $\begin{array}{l}-0.05(-0.14 \\
0.04)\end{array}$ & 0.251 \\
\hline \multirow[t]{4}{*}{ VFT-a } & $\begin{array}{l}\text { No-Diabetes }(n= \\
1226)\end{array}$ & Ref. & & Ref. & \\
\hline & Prediabetes $(n=2866)$ & $\begin{array}{l}-0.07(-0.13 \\
-0.01)\end{array}$ & 0.033 & $\begin{array}{l}-0.05(-0.11 \\
0.01)\end{array}$ & 0.101 \\
\hline & $\begin{array}{l}<5 y \text { Diabetes }(\mathrm{n}= \\
870)\end{array}$ & $\begin{array}{l}-0.14(-0.22 \\
-0.05)\end{array}$ & $0.001 *$ & $\begin{array}{l}-0.10(-0.17 \\
-0.02)\end{array}$ & 0.018 \\
\hline & $\begin{array}{l}\geq 5 y \text { Diabetes }(n= \\
889)\end{array}$ & $\begin{array}{l}-0.25(-0.33 \\
-0.16)\end{array}$ & $<.001^{*}$ & $\begin{array}{l}-0.18(-0.26 \\
-0.10)\end{array}$ & $<0.001 *$ \\
\hline \multirow[t]{4}{*}{ VFT-p } & $\begin{array}{l}\text { No-Diabetes }(n= \\
1227)\end{array}$ & Ref. & & Ref. & \\
\hline & Prediabetes $(n=2865)$ & $\begin{array}{l}-0.05(-0.12 \\
0.02)\end{array}$ & 0.149 & $\begin{array}{l}-0.03(-0.09 \\
0.03)\end{array}$ & 0.348 \\
\hline & $\begin{array}{l}<5 y \text { Diabetes }(\mathrm{n}= \\
870)\end{array}$ & $\begin{array}{l}-0.13(-0.21 \\
-0.04)\end{array}$ & $0.005^{\star}$ & $\begin{array}{l}-0.08(-0.16 \\
0.01)\end{array}$ & 0.060 \\
\hline & $\begin{array}{l}\geq 5 y \text { Diabetes }(n= \\
889)\end{array}$ & $\begin{array}{l}-0.23(-0.32 \\
-0.14)\end{array}$ & $<.001 *$ & $\begin{array}{l}-0.15(-0.23 \\
-0.07)\end{array}$ & $<0.001 *$ \\
\hline \multirow[t]{4}{*}{ TMT-A§ } & $\begin{array}{l}\text { No-Diabetes }(n= \\
1226)\end{array}$ & Ref. & & Ref. & \\
\hline & Prediabetes $(\mathrm{n}=2862)$ & $\begin{array}{l}-0.02(-0.08 \\
0.04)\end{array}$ & 0.512 & $\begin{array}{l}-0.03(-0.09 \\
0.03)\end{array}$ & 0.323 \\
\hline & $\begin{array}{l}<5 y \text { Diabetes }(n= \\
869)\end{array}$ & $0.08(0.01,0.16)$ & 0.037 & $0.05(-0.02,0.13)$ & 0.185 \\
\hline & $\begin{array}{l}\geq 5 y \text { Diabetes }(n= \\
886)\end{array}$ & $0.20(0.11,0.29)$ & <. $001 *$ & $0.15(0.06,0.23)$ & $0.001^{*}$ \\
\hline \multirow[t]{4}{*}{ TMT-B§ } & $\begin{array}{l}\text { No-Diabetes }(n= \\
1221)\end{array}$ & Ref. & & Ref. & \\
\hline & Prediabetes $(n=2859)$ & $0.01(-0.05,0.07)$ & 0.690 & $0.01(-0.06,0.06)$ & 0.994 \\
\hline & $\begin{array}{l}<5 y \text { Diabetes }(n= \\
866)\end{array}$ & $0.11(0.03,0.20)$ & $0.006^{*}$ & $0.08(0.01,0.16)$ & 0.039 \\
\hline & $\begin{array}{l}\geq 5 y \text { Diabetes }(n= \\
883)\end{array}$ & $0.24(0.15,0.32)$ & $<.001 *$ & $0.17(0.09,0.25)$ & $<0.001^{*}$ \\
\hline \multicolumn{6}{|c|}{$\begin{array}{l}\text { Abbreviations: }<5 y \text { diabetes, less than } 5 \text { years diabetes duration; } \geq 5 y \text { diabetes, more than } 5 \text { years } \\
\text { diabetes duration; GCF, Global Cognitive Function; MMSE, Mini-Mental State Examination; CDT, Clock } \\
\text { Drawing Test; DST-f, Digit Span Test forward section; DST-b, Digit Span Test backward section; VFT-a, } \\
\text { Verbal Fluency Test animal category; VFT-p, Verbal Fluency Test letter "p"; TMT-A, Trail Making Test } \\
\text { part A; TMT-B, Trail Making Test part B }\end{array}$} \\
\hline
\end{tabular}


Model 1: adjusted for sex, age (in years), intervention group, and center size (<250; 250-300, 300$400 ; \geq 400)$

Model 2: further adjusted for baseline education level (primary school; secondary school; college), civil status (single, divorced or separated; married; widower), physical activity (MET min/week), smoking habits (smoker; former smoker; never smoker), alcohol intake ( $\mathrm{g} /$ day, adding the quadratic term), 17point Mediterranean diet score, BMI $\left(\mathrm{kg} / \mathrm{m}^{2}\right)$, hypertension (yes/no), hypercholesterolemia (yes/no), and depressive symptomatology (yes/no)

Beta coefficients were estimated using linear regression models with robust standard errors to account for intracluster correlations

* Significant association after Benjamini-Hochberg correction

Additional file 1 shows odds ratio $(95 \% \mathrm{Cl})$ for cognitive impairment incidence after 2 years of follow-up in participants with normal cognitive performance at baseline by diabetes status. Compared with participants without diabetes, those with diabetes had a non-significant $34 \%(95 \% \mathrm{Cl} 0.96 ; 1.87)$ higher risk of cognitive impairment in the GCF Z-score, and a non-significant $30 \%(95 \% \mathrm{Cl} 1.01 ; 1.68)$ higher risk in the VFT-a test after the false discovery rate correction. No significant associations were found between diabetes status and cognitive impairment incidence in the rest of cognitive tests (Additional file 1: Supplemental Table 2).

Table 3 shows the association between baseline HOMA-IR (per one unit increment) and changes in cognitive Z-scores after 2 years of follow-up. Significant inverse associations between HOMA-IR and changes in cognitive Z-scores measured by GCF and the DST-f and DST-b tests were found (model 2). No significant associations between insulin resistance and changes in cognitive Z-scores were found in the MMSE, CDT, VFT-a, VFT-p and TMT-A tests. 
Table 3

Association between baseline HOMA-IR levels (per one unit increment) and changes in cognitive Z-scores

\begin{tabular}{|c|c|c|c|c|}
\hline \multirow[t]{2}{*}{ Z-scores } & \multicolumn{2}{|l|}{ Model 1} & \multicolumn{2}{|l|}{ Model 2} \\
\hline & $\beta(95 \% \mathrm{Cl})$ & P-value & $\beta(95 \% \mathrm{Cl})$ & P-value \\
\hline GCF $(n=4377)$ & $\begin{array}{l}-0.0140(-0.0217 \\
-0.0061)\end{array}$ & <. $001 *$ & $\begin{array}{l}-0.0094(-0.0164 \\
-0.0023)\end{array}$ & $0.009 *$ \\
\hline MMSE $(n=5180)$ & $-0.0040(-0.0120,0.0039)$ & 0.322 & $-0.0006(-0.0087,0.0075)$ & 0.884 \\
\hline $\operatorname{CDT}(n=5183)$ & $-0.0006(-0.0075,0.0064)$ & 0.868 & $-0.0006(-0.0077,0.0065)$ & 0.862 \\
\hline DST-f $(n=4560)$ & $\begin{array}{l}-0.0116(-0.0195 \\
-0.0037)\end{array}$ & $0.004^{\star}$ & $\begin{array}{l}-0.0091(-0.0170 \\
-0.0013)\end{array}$ & 0.023 \\
\hline DST-b $(n=4559)$ & $\begin{array}{l}-0.0106(-0.0184 \\
-0.0028)\end{array}$ & $0.007^{\star}$ & $\begin{array}{l}-0.0082(-0.0157 \\
-0.0006)\end{array}$ & 0.035 \\
\hline VFT-a $(n=5319)$ & $-0.0072(-0.0144,0.0001)$ & 0.051 & $-0.0050(-0.0121,0.0020)$ & 0.163 \\
\hline VFT-p $(n=5319)$ & $-0.0065(-0.0146,0.0015)$ & 0.111 & $-0.0042(-0.0115,0.0030)$ & 0.249 \\
\hline $\begin{array}{l}\text { TMT-A }(n= \\
\text { 5311)§ }\end{array}$ & $0.0070(-0.0008,0.0147)$ & 0.077 & $0.0040(-0.0037,0.0117)$ & 0.306 \\
\hline $\begin{array}{l}\text { TMT-B }(n= \\
5301) \S\end{array}$ & $0.0087(0.0014,0.0159)$ & 0.019 & $0.0060(-0.0007,0.0127)$ & 0.079 \\
\hline
\end{tabular}

Abbreviations: GCF, Global Cognitive Function; MMSE, Mini-Mental State Examination; CDT, Clock Drawing Test; DST-f, Digit Span Test forward section; DST-b, Digit Span Test backward section; VFT-a, Verbal Fluency Test animal category; VFT-p, Verbal Fluency Test letter " $p$ "; TMT-A, Trail Making Test part A; TMT-B, Trail Making Test part B

$\S$ Inverse neuropsychological assessment score

Participants with insulin treatment are excluded $(n=320)$

Model 1: adjusted for sex, age (in years), intervention group, and center size ( $<250 ; 250-300,300-$ $400 ; \geq 400)$

Model 2: further adjusted for baseline education level (primary school; secondary school; college), civil status (single, divorced or separated; married; widower), physical activity (MET min/week), smoking habits (smoker; former smoker; never smoker), alcohol intake (g/day, adding the quadratic term), 17point Mediterranean diet score, $\mathrm{BMI}\left(\mathrm{kg} / \mathrm{m}^{2}\right)$, hypertension (yes/no), hypercholesterolemia (yes/no), and depressive symptomatology (yes/no)

Beta coefficients were estimated using linear regression models with robust standard errors to account for intracluster correlations

* Significant association after Benjamini-Hochberg correction 
Table 4 presents the association between baseline $\mathrm{HbA} 1 \mathrm{c}$ levels (per one $\mathrm{mmol} / \mathrm{mol}$ increment) and 2year changes in cognitive Z-scores. An inverse association was observed between baseline $\mathrm{HbA1c}$ levels and the GCF score and the MMSE, VFT-a, VFT-p, TMT-A and TMT-B tests. No significant associations were found for the CDT and DST-f tests. 
Table 4

Association between baseline $\mathrm{HbA} 1 \mathrm{c}$ levels (per one $\mathrm{mmol} / \mathrm{mol}$ increment) and cognitive Z-scores changes

\begin{tabular}{|c|c|c|c|c|}
\hline \multirow[t]{2}{*}{ Z-scores } & \multicolumn{2}{|l|}{ Model 1} & \multicolumn{2}{|l|}{ Model 2} \\
\hline & $\beta(95 \% \mathrm{Cl})$ & P-value & $\beta(95 \% \mathrm{Cl})$ & P-value \\
\hline GCF $(n=4406)$ & $\begin{array}{l}-0.0085(-0.0115 \\
-0.0055)\end{array}$ & $\dot{0} 001 *$ & $\begin{array}{l}-0.0056(-0.0081 \\
-0.0030)\end{array}$ & $\dot{0} .001 *$ \\
\hline MMSE $(n=5162)$ & $\begin{array}{l}-0.0043(-0.0071 \\
-0.0015)\end{array}$ & $0.002^{\star}$ & $\begin{array}{l}-0.0029(-0.0055 \\
-0.0002)\end{array}$ & $0.035^{\star}$ \\
\hline $\operatorname{CDT}(n=5166)$ & $-0.0017(-0.0043,0.0009)$ & 0.210 & $-0.0007(-0.0032,0.0019)$ & 0.615 \\
\hline DST-f $(n=4601)$ & $-0.0030(-0.0061,0.0001)$ & 0.058 & $-0.0015(-0.0045,0.0015)$ & 0.330 \\
\hline DST-b $(n=4600)$ & $\begin{array}{l}-0.0042(-0.0072 \\
-0.0013)\end{array}$ & $0.005^{\star}$ & $-0.0023(-0.0051,0.0005)$ & 0.114 \\
\hline VFT-a $(n=5316)$ & $\begin{array}{l}-0.0071(-0.0099 \\
-0.0043)\end{array}$ & $\dot{0} .001^{*}$ & $\begin{array}{l}-0.0051(-0.0078 \\
-0.0024)\end{array}$ & $\dot{0} .001^{*}$ \\
\hline VFT-p $(n=5316)$ & $\begin{array}{l}-0.0087(-0.0118 \\
-0.0056)\end{array}$ & $\dot{0} 001^{*}$ & $\begin{array}{l}-0.0063(-0.0091 \\
-0.0035)\end{array}$ & $<.001^{*}$ \\
\hline $\begin{array}{l}\text { TMT-A }(n= \\
\text { 5307)§ }\end{array}$ & $0.0074(0.0045,0.0103)$ & $<.001 *$ & $0.0053(0.0025,0.0081)$ & $<.001 *$ \\
\hline $\begin{array}{l}\text { TMT-B }(n= \\
5296) \S\end{array}$ & $0.0072(0.0043,0.0100)$ & $\dot{0} .001 *$ & $0.0045(0.0019,0.0072)$ & $0.001^{*}$ \\
\hline \multicolumn{5}{|c|}{$\begin{array}{l}\text { Abbreviations: GCF, Global Cognitive Function; MMSE, Mini-Mental State Examination; CDT, Clock } \\
\text { Drawing Test; DST-f, Digit Span Test forward section; DST-b, Digit Span Test backward section; VFT-a, } \\
\text { Verbal Fluency Test animal category; VFT-p, Verbal Fluency Test letter "p"; TMT-A, Trail Making Test } \\
\text { part A; TMT-B, Trail Making Test part B }\end{array}$} \\
\hline \multicolumn{5}{|c|}{$\S$ Inverse neuropsychological assessment score } \\
\hline \multicolumn{5}{|c|}{ Missing data on $\mathrm{HbA}_{1 \mathrm{c}}(\mathrm{n}=633)$} \\
\hline \multicolumn{5}{|c|}{$\begin{array}{l}\text { Model 1: adjusted for sex, age (in years), intervention group, and center size }(<250 ; 250-300,300- \\
400 ; \geq 400)\end{array}$} \\
\hline \multicolumn{5}{|c|}{$\begin{array}{l}\text { Model 2: further adjusted for baseline education level (primary school; secondary school; college), civil } \\
\text { status (single, divorced or separated; married; widower), physical activity (MET min/week), smoking } \\
\text { habits (smoke; former smoker; never smoker), alcohol intake (g/day, adding the quadratic term), 17- } \\
\text { point Mediterranean diet score, BMI (kg/m²), hypertension (yes/no), hypercholesterolemia (yes/no), } \\
\text { and depressive symptomatology (yes/no) }\end{array}$} \\
\hline \multicolumn{5}{|c|}{$\begin{array}{l}\text { Beta coefficients were estimated using linear regression models with robust standard errors to account } \\
\text { for intracluster correlations }\end{array}$} \\
\hline \multicolumn{5}{|c|}{ * Significant association after Benjamini-Hochberg correction } \\
\hline
\end{tabular}


There were no significant interactions by sex, age, hypertension or BMI between the glycemic status (diabetes status, HOMA-IR, HbA1c and glycemic control/treatment) and changes in the GCF score (all $p$ > $0.05)$. An interaction by age was found between the diabetes status and changes in the GCF score $(P=$ 0.046). Compared to participants without diabetes, those aged $\leq 65$ years with prediabetes, and with diabetes $<5$-year and $\geq 5$-year duration showed a higher decline in the GCF score, whereas participants aged $>65$ years with prediabetes showed an increased performance in the GCF score. No associations were found between diabetes duration and the GCF score in participants aged $>65$ years.

\section{Diabetes control and treatment}

Additional file 1 shows the association between baseline glycemic control $(\mathrm{HbA} 1 \mathrm{c} \geq 57 \mathrm{mmol} / \mathrm{mol}$ or $<57$ $\mathrm{mmol} / \mathrm{mol}$ ) in participants with diabetes and 2-year changes in cognitive Z-scores. Compared to participants with good diabetes control, those with poor control showed a higher decrement in the VFT-p $(\beta=-0.13[95 \% \mathrm{Cl}-0.22 ;-0.04]$ ) test (model 2$)$. No associations between glycemic control and the rest of cognitive tests were found (Additional file 1: Supplemental Table 3).

Additional file 1 shows the association between baseline insulin treatment in participants with diabetes and changes in cognitive Z-scores. Compared to participants without insulin treatment, those with insulin treatment showed a significantly greater decrease in cognitive function measured by the GCF score and the DST-f, DST-b, VFT-a, VFT-p, TMT-A and TMT-B tests. No associations were observed for the remaining cognitive tests assessed (MMSE and CDT) (Additional file 1: Supplemental Table 4). Concerning oral glucose medication use, sulfonylureas treatment was no significant associated with an increase in the TMT-A ( $\beta=0.22$ [95\% Cl 0.07;0.38]) Z-score after Benjamini-Hockberg correction (Additional file 1: Supplemental Table 5). No significant associations were shown between the use of metformin or IDDP-4 and changes in cognitive Z-scores (Additional file 1: Supplemental Tables 6 and 7, respectively). When the associations between diabetes treatment and cognitive function were further adjusted by diabetes duration or glycemic control, the results remain similar (model 3 ).

No significant interactions by sex, age, hypertension, and BMI were observed between diabetes control or treatment and changes in the GCF score.

\section{Discussion}

To the best of our knowledge, this is the first prospective study investigating associations between glycemic status (diabetes status/control/treatment, and HOMA-IR and HbA1c biomarkers) and cognitive function as measured by a battery of neuropsychological tests in a same large cohort of older adults at high risk of cardiovascular disease. In this community-based population, compared to participants without diabetes, those with diabetes showed a higher decline in several cognitive performance measurements. Additionally, longer duration of diabetes was associated with greater decreases in scores of tests measuring processing speed and executive functions. Furthermore, poor diabetes control, the use 
of insulin treatment, and increases in HOMA-IR and $\mathrm{HbA} 1 \mathrm{c}$ were inversely associated with cognitive functioning over a 2-year period.

Our results concur with those of meta-analyses of prospective studies, suggesting a higher risk of cognitive decline in type 2 diabetes [6-8]. The mechanisms explaining these associations remains largely unknown. Several risk factors for cognitive dysfunction in diabetes have been reported, but each of them appears to have weak isolated effects $[28,29]$. In order to control for these potential confounding factors, we have adjusted our statistical models for several recognized confounders, such as hypertension or depression [28].

Our results are similar to those reported in other studies, suggesting a greater risk of cognitive decline in participants with type 2 diabetes, especially affecting executive functions $[5,8,30]$. Regarding the visuospatial function, discrepancies in longitudinal studies have been reported in case of individuals with type 2 diabetes [31, 32]. Nevertheless, a small effect size in this function was reported in a meta-analysis conducted in 2014 [30]. In our study, a non-significant inverse association between diabetes and the CDT test was also observed. However, longer follow-up of our population may be needed to observe a greater decline in this cognitive dimension. Concerning the memory function, we only assessed immediate verbal memory by the DST-f test. This cognitive function remained borderline inversely associated with the presence of diabetes. Additionally, our results did not show in participants with diabetes a decrease in working memory related to executive functioning measured by the DST-b. These results are in line with those reported in a recent meta-analysis, in which immediate (measured by the DST-f) and working memory (measured by the DST-b) were not affected in type 2 diabetes, while the rest of memory and executive function abilities were reduced [8].

Our results also show that, compared to participants without diabetes, those with diabetes had a borderline increased risk of developing a cognitive impairment as measured by the GCF score, even with only 2 years of follow-up. Meta-analyses including prospective studies showed cognitive impairment in participants presenting type 2 diabetes $[6,33]$. However, it was not usual to report short-time periods in the association between type 2 diabetes and cognitive function, and it may be the reason for the discrepancies showed between the aforementioned meta-analyses and our study.

As far as we know, no longitudinal studies have been conducted assessing associations between diabetes status and cognitive dysfunction, while also considering both the prediabetes status and the duration of diabetes. Longitudinal cohorts showed contradictory results regarding the effect that prediabetes has on cognition $[5,12,31,34]$, which can be explained by the different range of ages and sample sizes, the tests and cognitive domains assessed, and the length of follow-up.

The observed interaction of the GCF score with age in prediabetes has not been previously reported in the literature and cannot be explained by a specific mechanism. We cannot rule out that this interaction was a random finding and it is a result that requires further studies. Concerning diabetes duration, our results are in line with other longitudinal studies in which higher rates of cognitive decline were described in individuals with longer duration of diabetes $[5,12]$.

Page 22/30 
Several mechanisms have been suggested to explain the association between diabetes status and control with changes in cognitive functioning. Among them, insulin resistance, hyperglycemic excursions and glycemic control have received much attention. Insulin resistance linked to low-grade inflammation is a factor contributing to the onset of diabetes, that appears to play a key role in the cognitive impairment associated with obesity and diabetes, given the role that insulin has in the brain promoting neuronal survival and synaptic plasticity and inhibiting apoptosis and neuroinflammation [35]. In case of peripheral insulin resistance and type 2 diabetes, a decrease in insulin permeation through the blood-brain barrier was observed, leading to a smaller amount of insulin reaching the brain, thus impairing neuronal activation, and inducing changes in synaptic plasticity, neuronal apoptosis and neuroinflammation, all responsible of cognitive deterioration [35].

Longitudinal studies linking insulin resistance, as measured by HOMA-IR, and cognitive dysfunction showed discrepancies. In an older U.S. population with 8 years of follow-up, baseline HOMA-IR was not associated to changes in global cognitive function [36]. However, in surviving patients with coronary heart disease, baseline HOMA-IR was associated with subsequent poorer cognitive performance overall and on cognitive domains on the tests of memory, executive function and attention over a 15-year period [37]. Our results were in line with those of the latter study, as we also observed an inverse association between baseline HOMA-IR and changes in cognitive performance using the GCF score.

Another mechanism explaining the deleterious effect of diabetes on cognitive functioning is the hyperglycemic status and glycemic excursions. Increased $\mathrm{HbA} 1 \mathrm{c}$ levels or high levels of repeated glucose measurements through time have been linked to cognitive decline and an increased risk of dementia in people without diabetes [38]. In our study, no associations between $\mathrm{HbA1}$ c levels and changes in cognitive function were observed in participants without diabetes (data not shown). Nevertheless, when HbA1c was measured as a continuous variable, we found a negative association between $\mathrm{HbA} 1 \mathrm{c}$ and all cognitive tests measurements except in case of the CDT and the DSTs, being in line with recent studies $[34,36]$

When diabetes is established, increased $\mathrm{HbA} 1 \mathrm{c}$ levels have been linked to diabetes-associated cognitive decline and dementia, but the strength of these relationships is weak [11]. In our study, compared to participants with optimal diabetes control, those with poor control showed a higher 2-year decrease in cognitive performance measured by the VFT-p test, but this association was not observed in case of the GCF score and other cognitive assessments. Unlike other typical diabetic end-organ complications, no clear evidence exists that the increased risk of cognitive impairment can be attributed solely to hyperglycaemic excursions and glycaemic control [11]. For example, the ACCORD MIND trial [39], which compared intensive with standard treatment with the aim to lower $\mathrm{HbA} 1 \mathrm{c}$ in people with long-standing type 2 diabetes, found no association between the interventions and cognition.

Several other mechanisms have been implicated in diabetes-related cognitive decline and dementia. For example, type 2 diabetes has substantial adverse effects on blood vessels and the heart [40], leading to an increased risk of stroke and small cerebral vessel disease. Indeed, neuropathological studies also 
report an increased burden of cerebrovascular lesions, especially of lacunar type, in people with diabetes [41].

Observational studies have reported that some oral glucose-lowering medications may have potential beneficial or deleterious effects on cognition $[6,13]$. In our study, contrary to other results showing an improved cognitive function [13], no associations between metformin and cognition were observed, as well as is not found for IDDP-4 or sulfonylureas use. However, in line with findings of recent metaanalyses, insulin-treated participants showed higher cognitive decline than those not treated with insulin $[6,13]$. This could be explained because usually these individuals had a worse glycaemic control and higher risk of hypoglycaemia, a condition that has been clearly linked to cognitive decline and dementia risk $[42,43]$.

It is worth to mention that a strength of the present study is the novelty of being one of the largest population-based studies longitudinally exploring at the same time relationships between cognition and diabetes status, markers of glucose metabolism, and diabetes control and treatment in an older population at high cardiovascular risk, including a large sample size in each diabetes status categories. Nevertheless, the present findings should be considered in the context of some limitations. Firstly, although we adjusted the models for many potential confounding factors, there may be residual confounding factors not assessed, such as genetic susceptibility (APOE genotype). Unfortunately, genetic data was not available in all PREDIMED-Plus study population. Secondly, some of the explored associations might not be significant given that they have been explored in the context of a short period of time (2 years). Finally, our study has been conducted in older Mediterranean individuals with overweight/obesity and metabolic syndrome, and therefore we cannot extrapolate our results to other populations.

\section{Conclusions}

In conclusion, several glycemic dysregulations, such as insulin resistance measured by HOMA-IR, diabetes status, longer duration of diabetes, poor glycemic control, and insulin and sulfonylureas treatment were associated with more pronounced cognitive decline over 2 years in older individuals with overweight/obesity and metabolic syndrome. We also reported that participants with type 2 diabetes had a borderline increased risk of developing cognitive impairment as measured by the GCF score compared to those without diabetes.

\section{Abbreviations}

BMI, Body Mass Index

CDT, Clock Drawing Test

DST-b, Digit Span Test backward section 
DST-f, Digit Span Test forward section

GCF, Global Cognitive Function

HbA1c, glycated hemoglobin

HOMA-IR, Homeostasis Model Assessment of Insulin Resistance

IDDP-4, dipeptidyl peptidase-4 inhibitors

MMSE, Mini-Mental State Examination

TMT-A, Trail Making Test A section

TMT-B, Trail Making Test B section

VFT-a, verbal Fluency Test animals category

VFT-p, Verbal Fluency Test letter $p$ category

\section{Declarations}

\section{ETHICS APPROVAL AND CONSENT TO PARTICIPATE}

The ethical committees of the participating institutions approved the study protocol. Written informed consent for participation in the study was obtained from all the subjects.

\section{CONSENT FOR PUBLICATION}

All authors have read and approved the submission of the manuscript. The manuscript has not been published and is not being considered for publication elsewhere, in whole or in part, in any language. If the manuscript is accepted, we approve it for publication in Cardiovascular Diabetology.

\section{AVAILABILITY OF DATA AND MATERIALS}

There are restrictions on the availability of data for the PREDIMED-Plus trial, due to the signed consent agreements around data sharing, which only allow access to external researchers for studies following the project purposes. Requestors wishing to access the PREDIMED-Plus trial data used in this study can make a request to the PREDIMED-Plus trial Steering Committee chair:

predimed_plus_scommitte@googlegroups.com. The request will then be passed to members of the PREDIMED-Plus Steering Committee for deliberation.

COMPETING INTERESTS

J.S-S. serves on the board of the International Nut and Dried Fruit Council and receives grant support through this institution. He also served on the Executive Committee of the Instituto Danone, Spain, and on 
the Scientific Committee of the Danone International Institute. He has received research support from the Patrimonio Comunal Olivarero, Spain, and Borges S.A., Spain. He receives consulting fees or travel expenses from Eroski Foundation, the Instituto Danone, Spain, Mundipharma and Abbot Laboratories. E.R. reports grants, personal fees, non-financial support and other from California Walnut Commission and Alexion, personal fees, non-financial support and other from Ferrer International and Danone, and personal fees from Amarin, outside the submitted work. F.F-A reports consultation fees from Novo Nordisk and editor-in-Chief honoraria from Wiley. The rest of authors do not have relevant conflicts of interest to disclose.

FUNDING

This work was supported by the official Spanish Institutions for funding scientific biomedical research, CIBER Fisiopatología de la Obesidad y Nutrición (CIBEROBN) and Instituto de Salud Carlos III (ISCIII), through the Fondo de Investigación para la Salud (FIS), which is co-funded by the European Regional Development Fund (six coordinated FIS projects leaded by J.S-S. and J.Vi., including the following projects: Pl13/00673, Pl13/00492, PI13/00272, Pl13/01123, PI13/00462, Pl13/00233, PI13/02184, $\mathrm{Pl} 13 / 00728, \mathrm{Pl} 13 / 01090, \mathrm{PI} 13 / 01056, \mathrm{PI}$ 14/01722, PI14/00636, PI14/00618, PI14/00696, PI14/01206, PI14/01919, PI14/00853, PI14/01374, PI14/00972, PI14/00728, PI14/01471, PI16/00473, PI16/00662, $\mathrm{PI}$ 16/01873, PI16/01094, PI16/00501, PI16/00533, PI16/00381, PI16/00366, PI16/01522, PI16/01120, $\mathrm{PI} 17 / 00764, \mathrm{Pl} 17 / 01183, \mathrm{PI} 17 / 00855, \mathrm{PI} 17 / 01347, \mathrm{PI} 17 / 00525, \mathrm{PI} 17 / 01827, \mathrm{PI} 17 / 00532, \mathrm{PI} 17 / 00215$, $\mathrm{PI} 7 / 01441, \mathrm{Pl} 17 / 00508, \mathrm{PI} 7 / 01732, \mathrm{PI} 17 / 00926, \mathrm{PI} 19 / 00957, \mathrm{PI} 19 / 00386, \mathrm{PI} 19 / 00309, \mathrm{PI}$ 19/01032, $\mathrm{PI} 19 / 00576, \mathrm{PI}$ 19/00017, PI19/01226, PI19/00781, PI19/01560, PI19/01332, PI20/01802, PI20/00138, $\mathrm{PI} 20 / 01532, \mathrm{Pl} 20 / 00456, \mathrm{Pl} 20 / 00339, \mathrm{Pl} 20 / 00557, \mathrm{PI} 20 / 00886, \mathrm{PI} 20 / 01158)$; the Especial Action Project entitled: Implementación y evaluación de una intervención intensiva sobre la actividad física Cohorte PREDIMED-Plus grant to J.S-S.; the European Research Council (Advanced Research Grant 2014-2019; agreement \#340918) granted to M.Á.M-G.; the Recercaixa (agreement \#2013ACUP00194) grant to J.S-S.; grants from the Consejería de Salud de la Junta de Andalucía (PI0458/2013, PS0358/2016, PI0137/2018); the PROMETEO/2017/017 grant from the Generalitat Valenciana; the SEMERGEN grant; The Horizon 2020 PRIME study (Prevention and Remediation of Insulin Multimorbidity in Europe; grant agreement \#847879); J.J. holds the Miguel Servet-II contract (CPII19/00015) awarded by the Instituto de Salud Carlos III (co-funded by the European Social Fund "Investing in your future"); J.K. was supported by the 'FOLIUM' programme within the FUTURMed project from the Fundación Instituto de Investigación Sanitaria Illes Balears (financed by 2017annual plan of the sustainable tourism tax and at $50 \%$ with charge to the ESF Operational Program 2014-2020 of the Balearic Islands); A.A-S. received a postdoctoral grant from the Generalitat Valenciana (APOSTD/2020/164); C.G-M. receives a predoctoral grant from the University of Rovira i Virgili (2020PMF-PIPF-37); We thank CERCA Programme/Generalitat de Catalunya for institutional support and partial support was also provided by SLT006/17/00246, funded by the Department of Health of the Generalitat de Catalunya by the calls "Acció instrumental de programes de recerca orientats en l'àmbit de la recerca i la innovació en salut" and "Pla estratègic de recerca i innovació en salut (PERIS)"; J.S-S., senior author of this article, is partially supported by ICREA under the 
ICREA Academia program; None of the funding sources took part in the design, collection, analysis, interpretation of the data, or writing the report, or in the decision to submit the manuscript for publication.

\section{AUTHORS' CONTRIBUTIONS}

The principal PREDIMED-Plus investigators (M.A.M-G., J.S-S., D.C., J.A.M., A.M.A-G., J.W., J.V., D.R., J.L-M., R.E., F.J.T., J.L., L.S-M., A.B-C., J.A.T., V.M-S., X.P., M.D-R., P.M-M., J.Vi., C.V., L.D., E.R.) contributed to study concept and design and to data extraction from the participants. C.G-M., N.B-T., N.B., J.J. and J.S-S. performed the statistical analyses. C.G-M., and J.S-S. drafted the manuscript. All authors reviewed the manuscript for important intellectual content and approved the final version to be published.

\section{ACKNOWLEDGEMENTS}

The authors wish to thank the PREDIMED-Plus participants and staff for their engagement, as well as to the primary care centers involved in the study. We also thank the Cerca Programme of the Generalitat de Catalunya, and the CIBEROBN, CIBERESP and CIBERDEM initiatives of Instituto de Salud Carlos III in Spain, and the collaborators in the PRIME consortium for helpful input.

\section{References}

1. IDF Diabetes Atlas. 9th ed. Brussels: International Diabetes Federation; 2019.

2. Gregg EW, Sattar N, Ali MK. The changing face of diabetes complications. Lancet Diabetes Endocrinol. 2016;4(6):537-47.

3. World Health Organization. A report about dementia [Internet]. World Health Organization; 2020 [cited 2020 Nov 2]. Available from: https://www.who.int/news-room/fact-sheets/detail/dementia

4. Assuncao N, Sudo FK, Drummond C, De Felice FG, Mattos P. Metabolic syndrome and cognitive decline in the elderly: A systematic review. PLoS One. 2018;13(3).

5. Rawlings AM, Sharrett AR, Schneider ALC, Coresh J, Albert M, Couper D, et al. Diabetes in Midlife and Cognitive Change Over 20 Years. Ann Intern Med. 2014;161(11):785.

6. Xue M, Xu W, Ou Y-N, Cao X-P, Tan M-S, Tan L, et al. Diabetes mellitus and risks of cognitive impairment and dementia: A systematic review and meta-analysis of 144 prospective studies. Ageing Res Rev. 2019;55:100944.

7. Palta P, Schneider ALC, Biessels GJ, Touradji P, Hill-Briggs F. Magnitude of Cognitive Dysfunction in Adults with Type 2 Diabetes: A Meta-analysis of Six Cognitive Domains and the Most Frequently Reported Neuropsychological Tests Within Domains. J Int Neuropsychol Soc. 2014;20(3):278-91.

8. Sadanand S, Balachandar R, Bharath S. Memory and executive functions in persons with type 2 diabetes: a meta-analysis. Diabetes Metab Res Rev. 2016;32(2):132-42.

9. van den Berg E, Reijmer YD, de Bresser J, Kessels RPC, Kappelle LJ, Biessels GJ. A 4 year follow-up study of cognitive functioning in patients with type 2 diabetes mellitus. Diabetologia. 2010;53(1):5865. 
10. Moran C, Beare R, Wang W, Callisaya M, Srikanth V. Type 2 diabetes mellitus, brain atrophy, and cognitive decline. Neurology. 2019;92(8):e823-30.

11. Geijselaers SLC, Sep SJS, Stehouwer CDA, Biessels GJ. Glucose regulation, cognition, and brain MRI in type 2 diabetes: a systematic review. Lancet Diabetes Endocrinol. 2015;3(1):75-89.

12. Tuligenga RH, Dugravot A, Tabák AG, Elbaz A, Brunner EJ, Kivimäki M, et al. Midlife type 2 diabetes and poor glycaemic control as risk factors for cognitive decline in early old age: a post-hoc analysis of the Whitehall II cohort study. Lancet Diabetes Endocrinol. 2014;2(3):228-35.

13. Zhang Q-Q, Li W-S, Liu Z, Zhang H-L, Ba Y-G, Zhang R-X. Metformin therapy and cognitive dysfunction in patients with type 2 diabetes: A meta-analysis and systematic review. Medicine (Baltimore). 2020;99(10):e19378.

14. Martínez-González MA, Buil-Cosiales P, Corella D, Bulló M, Fitó M, Vioque J, et al. Cohort Profile: Design and methods of the PREDIMED-Plus randomized trial. Int J Epidemiol. 2019;48(2):387-388o.

15. Alberti KG, Eckel RH, Grundy SM, Zimmet PZ, Cleeman JI, Donato KA, et al. Harmonizing the Metabolic Syndrome. Circulation. 2009;120(16):1640-5.

16. Matthews DR, Hosker JP, Rudenski AS, Naylor BA, Treacher DF, Turner RC. Homeostasis model assessment: insulin resistance and $\beta$-cell function from fasting plasma glucose and insulin concentrations in man. Diabetologia. 1985;28(7):412-9.

17. American Diabetes Association. 2. Classification and Diagnosis of Diabetes: Standards of Medical Care in Diabetes-2021. Diabetes Care. 2021;44(Supplement 1):S15-33.

18. Schröder H, Fitó M, Estruch R, Martínez-González MA, Corella D, Salas-Salvadó J, et al. A Short Screener Is Valid for Assessing Mediterranean Diet Adherence among Older Spanish Men and Women. J Nutr. 2011;141(6):1140-5.

19. Elosua R, Marrugat J, Molina L, Pons S, Pujol E. Validation of the Minnesota Leisure Time Physical Activity Questionnaire in Spanish Men. Am J Epidemiol. 1994;39(12):1197-209.

20. Elosua R, Garcia M, Aguilar A, Molina L, Covas M-I, Marrugat J. Validation of the Minnesota Leisure Time Physical Activity Questionnaire in Spanish Women. Med Sci Sport Exerc. 2000 Aug;32(8):14317.

21. Beck AT, Ward CH, Mendelson M, Mock J, Erbaugh J. An Inventory for Measuring Depression. Arch Gen Psychiatry. 1961;4(6):561.

22. Ingraham LJ, Aiken CB. An empirical approach to determining criteria for abnormality in test batteries with multiple measures. Neuropsychology. 1996;10(1):120-4.

23. Blesa R, Pujol M, Aguilar M, Santacruz P, Bertran-Serra I, Hernández G, et al. Clinical validity of the "mini-mental state" for Spanish speaking communities. Neuropsychologia. 2001;39(11):1150-7.

24. del Ser Quijano T, García de Yébenes MJ, Sánchez Sánchez F, Frades Payo B, Rodríguez Laso Á, Bartolomé Martínez MP, et al. Evaluación cognitiva del anciano. Datos normativos de una muestra poblacional española de más de 70 años. Med Clin (Barc). 2004;122(19):727-40.

25. Ganguli M, Belle S, Ratcliff G, Seaberg E, Huff FJ, Von der Porten K, et al. Sensitivity and specificity for dementia of population-based criteria for cognitive impairment: The MoVIES project. Journals 
Gerontol. 1993;48(4):152-61.

26. de Waal T, Pannekoek J, Scholtus S. Handbook of Statistical Data Editing and Imputation. Hoboken,NJ: John Wiley \& Sons; 2011.

27. Benjamini Y, Hochberg Y. Controlling the False Discovery Rate: A Practical and Powerful Approach to Multiple Testing. J R Stat Soc Ser B. 1995;57(1):289-300.

28. Feinkohl I, Price JF, Strachan MWJ, Frier BM. The impact of diabetes on cognitive decline: potential vascular, metabolic, and psychosocial risk factors. Alzheimers Res Ther. 2015;7(1):46.

29. Mansur RB, Lee Y, Zhou AJ, Carmona NE, Cha DS, Rosenblat JD, et al. Determinants of cognitive function in individuals with type 2 diabetes mellitus: A meta-analysis. Ann Clin Psychiatry. 2018;30(1):38-50.

30. Monette MCE, Baird A, Jackson DL. A Meta-Analysis of Cognitive Functioning in Nondemented Adults with Type 2 Diabetes Mellitus. Can J Diabetes. 2014;38(6):401-8.

31. Marseglia A, Dahl Aslan AK, Fratiglioni L, Santoni G, Pedersen NL, Xu W. Cognitive Trajectories of Older Adults With Prediabetes and Diabetes: A Population-Based Cohort Study. Journals Gerontol Ser A. 2018;73(3):400-6.

32. Wennberg AMV, Hagen CE, Gottesman RF, Zipunnikov V, Kaufmann CN, Albert MS, et al. Longitudinal association between diabetes and cognitive decline: The National Health and Aging Trends Study. Arch Gerontol Geriatr. 2017;72:39-44.

33. Zhang X, Jiang X, Han S, Liu Q, Zhou J. Type 2 Diabetes Mellitus Is Associated with the Risk of Cognitive Impairment: a Meta-Analysis. J Mol Neurosci. 2019;68(2):251-60.

34. Zheng F, Yan L, Yang Z, Zhong B, Xie W. HbA1c, diabetes and cognitive decline: the English Longitudinal Study of Ageing. Diabetologia. 2018;61(4):839-48.

35. Karvani M, Simos P, Stavrakaki S, Kapoukranidou D. Neurocognitive impairment in type 2 diabetes mellitus. Hormones. 2019;18(4):523-34.

36. Ganguli M, Beer JC, Zmuda JM, Ryan CM, Sullivan KJ, Chang CH, et al. Aging, Diabetes, Obesity, and Cognitive Decline: A Population-Based Study. J Am Geriatr Soc. 2020;68(5):991-8.

37. Lutski M, Weinstein G, Goldbourt U, Tanne D. Insulin Resistance and Future Cognitive Performance and Cognitive Decline in Elderly Patients with Cardiovascular Disease. J Alzheimer's Dis. 2017;57(2):633-43.

38. Crane PK, Walker R, Hubbard RA, Li G, Nathan DM, Zheng H, et al. Glucose Levels and Risk of Dementia. N Engl J Med. 2013;369(6):540-8.

39. Erus G, Battapady H, Zhang T, Lovato J, Miller ME, Williamson JD, et al. Spatial Patterns of Structural Brain Changes in Type 2 Diabetic Patients and Their Longitudinal Progression With Intensive Control of Blood Glucose. Diabetes Care. 2015;38(1):97-104.

40. Biessels GJ, Despa F. Cognitive decline and dementia in diabetes mellitus: mechanisms and clinical implications. Nat Rev Endocrinol. 2018;14(10):591-604. 
41. Abner EL, Nelson PT, Kryscio RJ, Schmitt FA, Fardo DW, Woltjer RL, et al. Diabetes is associated with cerebrovascular but not Alzheimer's disease neuropathology. Alzheimer's Dement. 2016;12(8):882-9.

42. Whitmer RA. Hypoglycemic Episodes and Risk of Dementia in Older Patients With Type 2 Diabetes Mellitus. JAMA. 2009;301(15):1565-72.

43. Lin C-H, Sheu WHH. Hypoglycaemic episodes and risk of dementia in diabetes mellitus: 7-year followup study. J Intern Med. 2013;273(1):102-10.

\section{Supplementary Files}

This is a list of supplementary files associated with this preprint. Click to download.

- Additionalfile1.docx 